\title{
Direct observation of photoionization dynamics in solution phase induced by femtosecond two- photon excitation
}

\author{
Masafumi Koga*, Yusuke Yoneda, Hikaru Sotome, and Hiroshi Miyasaka \\ Graduate School of Engineering Science, Osaka University, 1-3 Machikaneyama, Toyonaka, Japan
}

\begin{abstract}
In solution phase, the solute can be photo-ionized in the lower excitation energy than its ionization potential in gas phase. Therefore, the specific interaction is expected to be exist between the surrounding media and higher excited $\left(\mathrm{S}_{\mathrm{n}}\right)$ state of the solute. In order to elucidate such polarization effect of solvent on the photoionization process, femtosecond double-pulse excitation was applied to direct detection of low-energy photoionization dynamics of a phenylenediamine derivative in solution phase. From the results of the transient absorption change, in polar solvent, it is clearly indicated that photoionization does not proceed directly from the $\mathrm{S}_{\mathrm{n}}$ state, but through specific intermediate state. Moreover,
\end{abstract}

\section{Introduction}

Photoionization is one of the simplest and most important primary processes in photochemical reactions. In the solution phase, polarization of surrounding media has been considered to play crucial roles in photoionization processes through the solvation. That is, stabilization of cation and ejected electron by the solvation has been conventionally considered to induce the ionization in the solution phase with the excitation energy lower than the ionization potential in the gas phase $[1,2]$. Although a number of investigations using time-resolved spectroscopies have been so far applied to the detection of photoionization dynamics [1-3], most of them discussed the time evolution after the ionization. Ultrafast time scales of the electron ejection still inhibit the clear elucidation of the real-time observation of "electron ejection" in solutions. Here, in order to fully elucidate the real-time dynamics and mechanism of the ejection of electron from the solute surrounded by solvent molecules, we have conducted femtosecond transient absorption measurements under the sequential twophoton excitation condition.

\section{Experimental}

$N, N, N^{\prime}, N^{\prime}$-tetramethyl-p-phenylenediamine (TMPD) was used as a solute. The absorption spectrum of TMPD in acetonitrile and relative fluorescence quantum yield of TMPD are shown in Fig. 1a. In acetonitrile and ethanol, the fluorescence quantum yield of

\footnotetext{
*Corresponding author: koga@laser.chem.es.osaka-u.ac.jp
} 
TMPD decreases in the shorter wavelength region than $280 \mathrm{~nm}$ but it keeps an almost constant value in $n$-hexane. These results strongly suggest that photoionization effectively takes place through higher excited states in polar acetonitrile and ethanol solution.

(a)

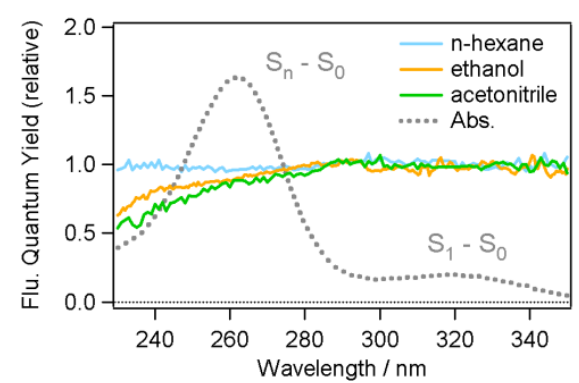

(b)

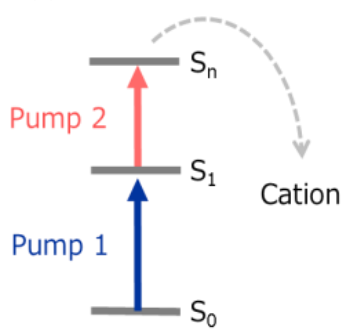

Fig. 1. (a) Steady-state absorption spectrum of TMPD in acetonitrile (grey dashed). Relative fluorescence quantum yield of TMPD in acetonitrile (orange line) and in $n$-hexane (blue line) normalized at $340 \mathrm{~nm}$ were also shown. (b) Schematic diagram of photoionization via higher excited states induced by femtosecond double-pulse excitation.

In order to directly elucidate photoionization via higher excited states, we constructed femtosecond transient absorption (TA) spectroscopy system under double-pulse excitation, as described in Fig. 1b. The output of a femtosecond Ti:sapphire laser was amplified by using a regenerative amplifier and the amplified $800-\mathrm{nm}$ pulse was divided into two pulses. One was introduced into an optical parametric amplifier (OPA) system and converted to an 1180-nm pulse, which was focused into a 2-mm $\mathrm{CaF}_{2}$ plate to generate white-light continuum covering the wavelength regime from 400 to $1000 \mathrm{~nm}$. This white light was used as a probe pulse. The other $800-\mathrm{nm}$ pulse was guided into non-collinear OPA (NOPA) system which was tuned at $680 \mathrm{~nm}$ and its output was frequency-doubled with a $50-\mu \mathrm{m}$ BBO crystal. The second harmonic $(340 \mathrm{~nm})$ was resonant with the $\mathrm{S}_{1} \leftarrow \mathrm{S}_{0}$ transition of TMPD and was used as the first pump pulse (Pump 1). The remaining 680-nm pulse was resonant with the $S_{n} \leftarrow S_{1}$ transition as shown in Fig. 2a and used as the second pump pulse (Pump 2). Pump 2 was introduced into the sample 10 ps after Pump 1 and the delay time $(\Delta t)$ is defined as the time interval between Pump 2 and probe pulses.

\section{Results and discussion}

Transient absorption spectra of TMPD in ethanol solution under the double-pulse excitation condition are shown in Figure 2a. Immediately after the irradiation of the pump2 (at $0 \mathrm{ps}$ in the figure), positive bands at $450-630 \mathrm{~nm}$ and $800-950 \mathrm{~nm}$ and negative band at 630-800 $\mathrm{nm}$ appeared. The negative band is safely ascribed to the bleaching of the $\mathrm{S}_{1}$ state, because this band has the negative peaks at 680 and $740 \mathrm{~nm}$ which are in good agreement with $S_{n} \leftarrow S_{1}$ absorption of TMPD in ethanol [3] . The positive signal around 450-630 nm shows the rapid decay and almost disappeared within $300 \mathrm{fs}$. Because this band appears within the instrumental response time, this absorption is due to the initially formed higher excited state. After the decay of the $S_{n} \leftarrow S_{1}$ absorption in sub-ps time region, a new positive band gradually grows up around $430-630 \mathrm{~nm}$ in the picosecond time regime. The absorption maxima of this absorption at 560 and $610 \mathrm{~nm}$ are in good agreement with those of TMPD radical cation [4]. These results indicate that appearance of TMPD cation is in the picosecond time scale in ethanol solution and it takes a rather long time after the excitation into the higher excited state. 
Figure $2 \mathrm{~b}$ and $2 \mathrm{c}$ show time traces of normalized transient absorbance integrated from 450 to $630 \mathrm{~nm}$ in ethanol together with that in $n$-hexane. The signal in ethanol can be reproduced with a single exponential decay with a time constant of $70 \mathrm{fs}$, which is shorter than that in $n$-hexane (200 fs). Because no ionization occurs in $n$-hexane, the shorter lifetime of the $S_{n}$ state in ethanol indicates that an additional process occurs from the $S_{n}$ state in ethanol. On the other hand, the new positive band with maxima at 560 and $610 \mathrm{~nm}$, which are clearly ascribed to TMPD radical cation, appears in the time scale of several ps in ethanol. The formation of the cation is considerably slower than the lifetime of the $S_{n}$ state, strongly indicating that the photoionization does not take place directly from the higher excited state but via a specific intermediate. Moreover, the formation time constant of the cation, $5.8 \mathrm{ps,}$ is analogous to the solvation time in ethanol solution [5]. This agreement strongly suggests shat ionization process is induced by solvation of the intermediate state produced from the $\mathrm{S}_{\mathrm{n}}$ state.

(a)

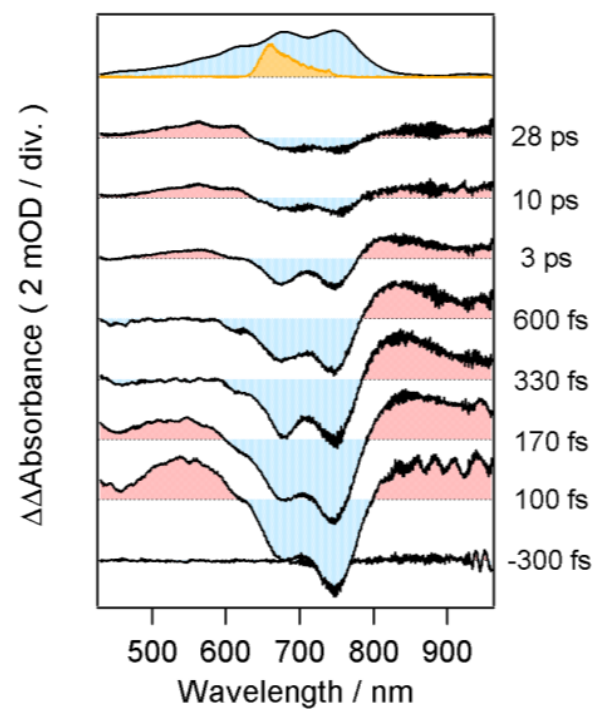

(b)

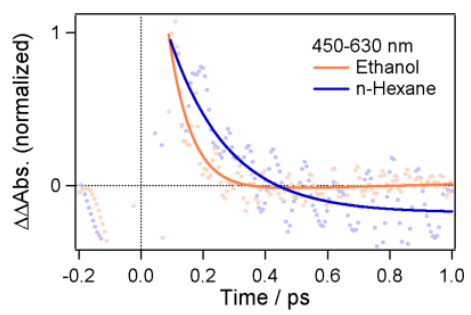

(c)

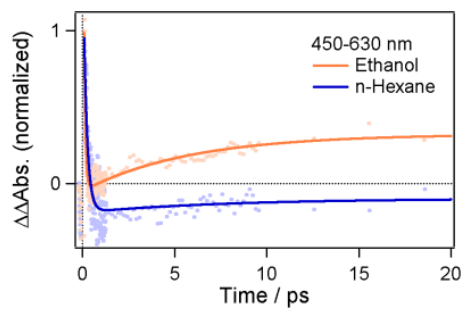

Fig. 2. (a) $\Delta \Delta$ Absorption spectra of TMPD in ethanol solution excited with pump1 (340 nm, $320 \mathrm{~nJ})$ and pump2 $(680 \mathrm{~nm}, 740 \mathrm{~nJ})$. The delay time was a time interval between pump2 and probe pulses. The delay of pump 1 was fixed to be -10 ps. The spectrum of pump 2 (orange) and transient absorption spectrum observed at $10 \mathrm{ps}$ after pump 1 irradiation (blue) are shown at the top of the figure. $(b, c)$ Time profiles of transient absorbance of TMPD in ethanol (orange lines) and $n$-hexane (blue lines) averaged over $450-630 \mathrm{~nm}$.

\section{References}

1. M. Ottolenghi, Chem. Phys. Lett. 12, 339 (1971)

2. J. A. Kloepfer, V.H. Vilchiz, V. A. Lenchenkov, A. C. Germaine, and S. E. Bradforth J. Chem. Phys. 113, 6288 (2000)

3. Y. Hirata, and N. Mataga J. Phys. Chem. 87, 3190 (1983)

4. A. C. Albrecht Acc. Chem. Res. 3, 238 (1970)

5. M. L. Horng, J. A. Garadecki, A. Papazyan, and M. Maroncelli J. Phys. Chem. 99, 17311 (1995) 